\section{KRAS wild-type tumors benefit from cetuximab}

A randomized, multicenter trial has shown that first-line treatment with cetuximab and the irinotecan-containing chemotherapy regimen FOLFIRI reduces the risk of progression in patients with metastatic colorectal cancer, but this benefit was limited to patients with wild-type KRAS tumors. Colorectal cancer is the third most common cancer in the world and almost $25 \%$ of patients present with metastatic disease. The anti-EGFR monoclonal antibody, cetuximab, is effective as a single agent or in combination with irinotecan-based therapies for patients with metastatic colorectal cancer. Mutations in KRAS have been shown to be correlated with a worse outcome in patients with colorectal cancer treated with the anti-EGFR monoclonal antibodies cetuximab and panitumumab.

A multicenter, randomized trial examined the efficacy and safety of FOLFIRI alone or in combination with cetuximab in patients with metastatic colorectal cancer, and assessed associations between KRAS status and response to cetuximab. The primary end point was progression-free survival, and secondary end points included overall survival, overall response rate and safety analysis. In total, 1,198 patients were randomly assigned to the different treatment arms in a 1:1 ratio. The risk of progression was reduced by $15 \%$ in the cetuximab and FOLFIRI arm compared with the FOLFIRI-only arm. The median progression-free survival time was higher in the combination arm than the FOLFIRI arm (8.9 months versus 8.0 months). No significant difference was demonstrated in the overall survival times between arms.

KRAS mutation data were available for samples obtained at baseline for 540 patients. More patients in the FOLFIRIonly group had wild-type KRAS tumors. There was a significant association between wild-type KRAS mutational status and response to cetuximab $(P=0.03)$. The risk of progression-free survival and overall survival, however, was not significantly affected by KRAS status. The risk of progression for patients receiving cetuximab was considerably reduced in patients with wild-type KRAS tumors, as highlighted by a 0.68 hazard ratio compared with 1.07 in patients with mutant KRAS tumors. The median progression-free survival rates were longer for patients with wild-type KRAS compared to those with the mutated gene. The overall incidence of adverse events was $26 \%$ for patients who received cetuximab and FOLFIRI and 19.3\% for those treated with FOLFIRI alone. The incidence of grade 3 skin reactions and acne-like rash, infusion-related

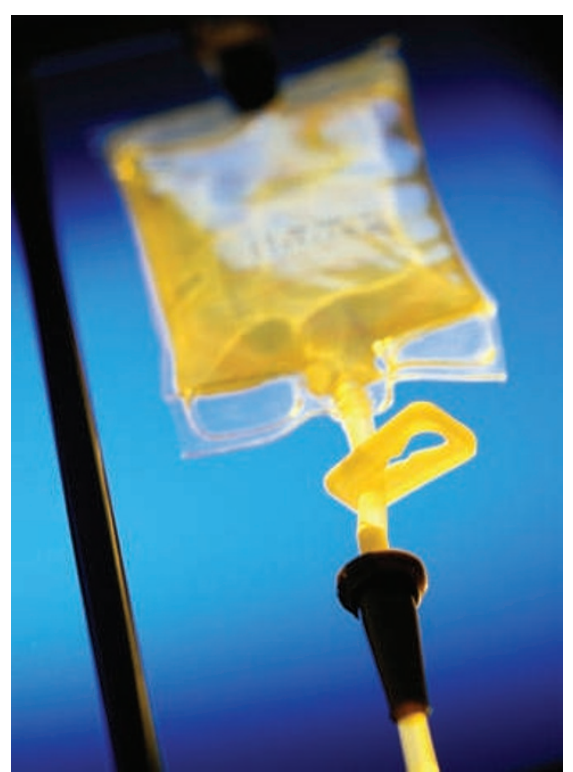

reactions and diarrhea were higher in the combination arm compared with the FOLFIRI arm.

"This trial provides confirmation that, as compared with FOLFIRI alone, cetuximab plus FOLFIRI reduces the risk of progression of metastatic colorectal cancer when used as first-line treatment, and that this benefit is seen mainly in patients with wild-type KRAS tumors," the authors conclude.

Lisa Hutchinson

Original article Van Cutsem, E. et al. Cetuximab and chemotherapy as initial treatment for metastatic colorectal cancer. N. Engl. J. Med. 360, 1408-1417 (2009). 